\title{
Inefficacy of autologous bone marrow concentrate in stage three osteonecrosis: a randomized controlled double-blind trial
}

\author{
Jean-Philippe Hauzeur ${ }^{1,2}$ (D) Viviane De Maertelaer ${ }^{3}$ - Etienne Baudoux ${ }^{4}$. \\ Michel Malaise $^{1} \cdot$ Yves Beguin $^{4} \cdot$ Valérie Gangji $^{2}$
}

Received: 13 May 2017 / Accepted: 21 September 2017 /Published online: 7 October 2017

(C) SICOT aisbl 2017

\begin{abstract}
Purpose The fracture stage of non-traumatic osteonecrosis (ON stage 3 ) of the femoral head (ONFH) has an unfavourable prognosis frequently requiring total hip replacement (THR). The percentage could be lowered after core decompression. In earlier non-fracture ON stages, implantation of autologous bone marrow aspirate concentrate (BMAC) improved the effect of core decompression. The purpose was to evaluate the effect of BMAC in addition to core decompression in stage 3 ONFH.

Methods A double blind RCT was conducted comparing two groups: core decompression plus saline injection or core decompression plus BMAC implantation. Both patients and assessors were blinded to the treatment assignments. Evaluations were done at baseline, three, six, 12, and 24 months, including pain (VAS), WOMAC, side-effects, radiological evolution including ARCO subclassifications, together with possible THR requirement. The primary endpoint was the need for THR. The second endpoints included the
\end{abstract}

Jean-Philippe Hauzeur

jean-philippe@hauzeur.org

Viviane De Maertelaer

vdemaert@ulb.ac.be

Etienne Baudoux

e.baudoux@chu.ulg.ac.be

Michel Malaise

michel.malaise@chu.ulg.ac.be

Yves Beguin

yves.beguin@chu.ulg.ac.be clinical symptoms such as pain and functional ability and the progression of the ON lesions as well as the appearance of osteoarthritis features (ARCO stage 4). Both groups included 23 hips (19 patients).

Results No differences were found between the groups for THR requirements, clinical tests, and radiological evolution. In both groups, 15/23 hips needed THR. The radiological evolution of the ONFH lesions in term of location, extension, surface collapse, and dome depression was moderate in both groups and was not correlated with the need of THR.

Conclusions Implantation of BMAC after core decompression did not produce any improvement of the evolution of ONFH stage 3.

Level of evidence I.

Keywords Osteonecrosis $\cdot$ Femoral head $\cdot$ Bone marrow aspirate concentrate $\cdot$ Cell therapy $\cdot$ Mesenchymal stem cells . Hip

Valérie Gangji

Valerie.Gangji@erasme.ulb.ac.be

1 Department of Rheumatology, CHU de Liège, University of Liège, B 4000 Liège, Belgium

2 Department of Rheumatology and Physical Medicine, Hôpital Erasme, Université Libre de Bruxelles, 808 Route de Lennik, 1070 Brussels, Belgium

3 Department of Biostatistics and Medical Informatics \& IRIBHM, School of Medicine, Université Libre de Bruxelles, ULB, 808 route de Lennik, 1070 Brussels, Belgium

4 Laboratory of Cell and Gene Therapy, CHU de Liège, University of Liège, Liège, Belgium 


\section{Introduction}

Aseptic non-traumatic osteonecrosis of the femoral head (ONFH) is a painful disorder which leads in $80 \%$ of cases to a total hip replacement (THR) [1]. Glucocorticoids and alcohol abuse are the most frequent risk factors for ONFH in Caucasians [2].

The treatment of ONFH, particularly in fracture stages, remains challenging. Two specific features of ONFH emphasize the importance of developing a conservative surgical therapeutic approach since prosthetic replacements rarely last for their lifetime and could be required for more than one joint. Firstly, patients suffering from ONFH are young. In a recent ONFH cohort study the mean age was 48.4 years with the youngest being 19 years old [3]. Secondly, patients suffering from ONFH have a high risk of multiple surgical procedures. Indeed, multiple ON lesions are frequent: bilateral lesions were found in $63 \%$ of the patients and $11 \%$ had multifocal ON (more than 2 ON sites) [3]. Core decompression of the femoral head is the most widespread conservative surgical procedure proposed to treat ONFH. Although this procedure has already been employed for more than five decades [4], its efficacy remains controversial $[5,6]$. The success rate for core decompression is even worse when a subchondral fracture (47\% in ARCO stage 3) is present compared to the precollapse stages (85\% in ARCO stage 1 and $65 \%$ in ARCO stage 2) $[1,7]$.

Recent publications suggested that ON might be a disease of bone cells and mesenchymal stem cells (MSC) $[8$, 9]. The activity levels together with the number of MSC in the bone marrow is depressed in patients with ONFH [8]. The replication capacity of osteoblastic cells is decreased in the proximal femur of patients with ONFH [9]. The apoptosis of osteoblasts and osteocytes is increased especially in the so-called creeping substitution surrounding the necrotic region [10]. These findings raised the possibility that bone marrow containing MSC implanted into the necrotic lesion of the femoral head could be useful. A two year prospective controlled study showed that, in unfractured ONFH cases, the implantation of autologous bone marrow aspirate concentrate (BMAC) into the necrotic lesion through a core decompression procedure was more effective than core decompression alone in improving pain and hip function and reducing the number of hips that progressed to subchondral fracture [11]. The course of the disease over 24 months was deemed satisfactory in $90 \%$ of the hips in the BMAC group compared to $37.5 \%$ in the control group. This positive effect was confirmed in a five year follow-up study [12].

The natural evolution of ON stage 3 seems poor, with $87 \%$ of cases requiring THR, which may be reduced to $53 \%$ after core decompression [1]. A beneficial effect - reduction of both functional evolution and need for THR - has also been reported in fracture ONFH [13]. The purpose of the present study was, therefore, to evaluate the effect of the addition of BMAC implanted in the necrotic lesion in comparisonn with a core decompression alone in ARCO stage 3 ONFH. We initiated a randomized controlled trial (RCT) on the effect of BMAC implantation into the necrotic lesion of fracture stages ONFH using the same technique as previously described [14].

\section{Patients and methods}

The study was conducted in two university centers: Hôpital Erasme, Université de Bruxelles and CHU de Liège, University of Liège. Osteonecrosis was diagnosed by X-rays and MRI for each case [15]. The ON staging used was the ARCO classification system using X-rays and MRI [16, 21].

The primary objective was to confirm, in ARCO stage 3 ONFH, the BMAC injection's effect on the need for a THR.

The secondary objectives were to evaluate the advantage produced by BMAC concerning pain, functional ability, progression of ON lesions in terms of location, extension, surface collapse, dome depression evolution, and appearance of osteoarthritis features (evolution to ARCO stage 4).

The inclusion criteria were patients aged 18 years or older suffering from ARCO stage 3 non-traumatic ONFH with a surface collapse lower than $30 \%$ of the entire articular surface together with a dome depression of no more than $4 \mathrm{~mm}$, and who had signed the written consent form. The exclusion criteria were evidence of malignant disorder in the past five years, contraindication to undergo an MRI, or a positive serological test for HIV, hepatitis B or hepatitis C.

\section{Randomization}

A centralized assignment to treatment groups was performed for the two centres. The subject under investigation was patient's hip. We therefore randomly allocated hips to a core decompression procedure associated with BMAC implantation (BMAC group) or saline implantation (control group). In case of eligibility, hips were allocated through a randomization process to one of the two study arms, with a ratio $1: 1$. A randomization list was generated, using random permuted blocks of two letters. Randomization was adapted for bilateral lesions because the bone marrow aspiration volume had to be limited for ethical reasons and MSC numbers decrease in larger bone marrow collection volumes [17] When the two hips (of the same patient) affected with ONFH were enrolled in the study, the right hip was assigned to the treatment arm from the first available envelop. The other hip was then assigned to the other treatment arm, using the next available envelop with said assignment, in order to ensure exactly equal treatment numbers as specified in the randomization code.

As proof-of-concept study, an optimal sample size was impossible to calculate. No formal sample size calculation 
was performed. Sixty subjects (hips) were planned to be enrolled. This number was considered sufficient to document the efficacy and safety.

This study was a double-bind study: participants and outcome assessor (clinicians and radiologists) were unaware of which treatment assignment was which. Treating clinicians were not masked to treatment assignment.

\section{Core decompression and BMAC procedures}

The procedures were previously published [14, 18]. Under general anaesthesia, a volume of $400 \mathrm{~mL}$ of bone marrow was harvested from the posterior iliac crests and was sorted on a Spectra cell separator $(777,006,300$; Cobe, Lakewood, Colorado) and concentrated to a final volume of $50 \mathrm{ml}$. During the same general anaesthesia, a special $4 \mathrm{~mm}$ trephine was inserted under control of a fluoroscopic view through the great trochanter, the neck, and the femoral head in the necrotic lesion. Then the BMAC (BMAC group) or saline $50 \mathrm{ml}$ (control group) was injected into the necrotic region. To avoid any leakage, a Gelfoam ${ }^{\circledR}$ absorbable gelatin sponge (Pharmacia \& Upjohn Company, Kalamazoo, MI 49001, USA) was pushed through the trephine to close the hole. In the control group, a sham skin incision was done at the site of bone marrow harvesting. So, the patients were unaware of the treatment received.

\section{Clinical evaluation}

Two investigators, unaware of the treatment group assignments, performed all the post-operative outcome assessments. Patients were assessed at baseline, three, six, 12, and 24 months. Patients' assessments of pain included a visual analogue scale (VAS) from $0 \mathrm{~mm}$ (no pain) to $100 \mathrm{~mm}$ (severe pain) [19]. Symptoms were also assessed by the subscales A and $\mathrm{B}$ (both dedicated to the hip status) of the WOMAC score [20]. In the case of hip bilateral involvement, patients were asked to complete a separate questionnaire for each hip. During each visit, safety was assessed by recording adverse events and serious adverse events. The decision to possibly turn to THR when the treatment had been insufficient in controlling pain and/or disability, was discussed with the patient at the end of each assessment session. The final decision was taken according to the patient's own wishes.

\section{Radiological evaluation}

Anteroposterior and frog leg view weight-bearing radiographs and an MRI study were taken at baseline, three, six, 12, 18, and 24 months. Radiological progression of ONFH was measured by reference to the ARCO staging [16, 21] For location and quantification of ON lesion, the ARCO subclassifications were used $[16,21]$. The location of the $\mathrm{ON}$ lesion under a weight-bearing dome of the acetabulum was $\mathrm{A}=$ medial, $\mathrm{B}=$ central, $\mathrm{C}=$ lateral; the extension of the lesion (a calculation of the area of femoral head involvement) was: $\mathrm{A}=<15 \%$, $\mathrm{B}=15-30 \%, \mathrm{C}=>30 \%$. The surface collapse extension was, after selection of the most prominent view, expressed as a percentage of the entire articular surface: $\mathrm{A}=<15 \%$ involvement, $\mathrm{B}=15-30 \%$ involvement, $\mathrm{C}=>30 \%$ involvement. The dome depression was expressed as $\mathrm{A}=\mathrm{a}$ depression of less than $2 \mathrm{~mm}, \mathrm{~B}=2-4 \mathrm{~mm}$, and $\mathrm{C}=$ more than $4 \mathrm{~mm}$. A single reader, unaware of the treatment assignments, analyzed all radiographs and MRI.

\section{Statistical methods}

Continuous variables were given by their mean \pm SEM; their means were compared between two independent groups with Student t-tests, or with Welch tests in the case of variance heterogeneity. Qualitative variables were given by their proportions and were compared between independent groups with Fisher's exact chi-square tests.

All statistical analyses were performed using the statistical software IBM-SPSS-V22.0. A statistically significant effect was considered as soon as the $p$-value was lower than 0.05 .

\section{Results}

The cohort formation is summarized in the CONSORT flow diagram (Fig. 1). We screened 60 hips from 46 patients (14 patients with bilateral stage $3 \mathrm{ON}$ ). We could randomize 52 hips/42 patients (ten with bilateral stage $3 \mathrm{ON}$ ) in the two groups. Finally, we were able to evaluate 23 hips from 19 patients (eight with bilateral stage $3 \mathrm{ON}$ ) in the BMAC group and 23 hips from 19 patients (eight with bilateral stage $3 \mathrm{ON}$ ) in the control group.

The baseline demographic characteristics are presented in Table 1. No statistical differences between both groups were found in terms of gender, age, BMI, side of unilateral ON, duration of symptoms, bilateral and multifocal ON presentations, risk factors, pain, and WOMAC scoring. The hips displayed similar fracture ARCO stage 3 ONFH in terms of location and extension of ON lesions, surface collapse, and dome depression.

The BMAC had a final volume of $48.33 \pm 1.16 \mathrm{ml}$. The mean number of nucleated cells injected was $3.46 \pm 0.36 \times 10^{9}$ including $1.5 \pm 0.28 \% \mathrm{CD} 34^{+}$. The mean number of Fibroblast colony forming units (CFU-F) was $19.45 \pm 3.51 \times 10^{6}$ nucleated cells.

At the 24-month follow-up, no difference was found between groups of either the primary or secondary endpoints (Table 2).

The primary objective was to confirm the effect of BMAC on the need for a THR. In the BMAC group 15 hips and in the control group 23 hips needed a THR $(p=1.000)$. The time to 
Fig. 1 CONSORT flow diagram for stage 3 ONFH BMAC versus core trial: hips (patients)

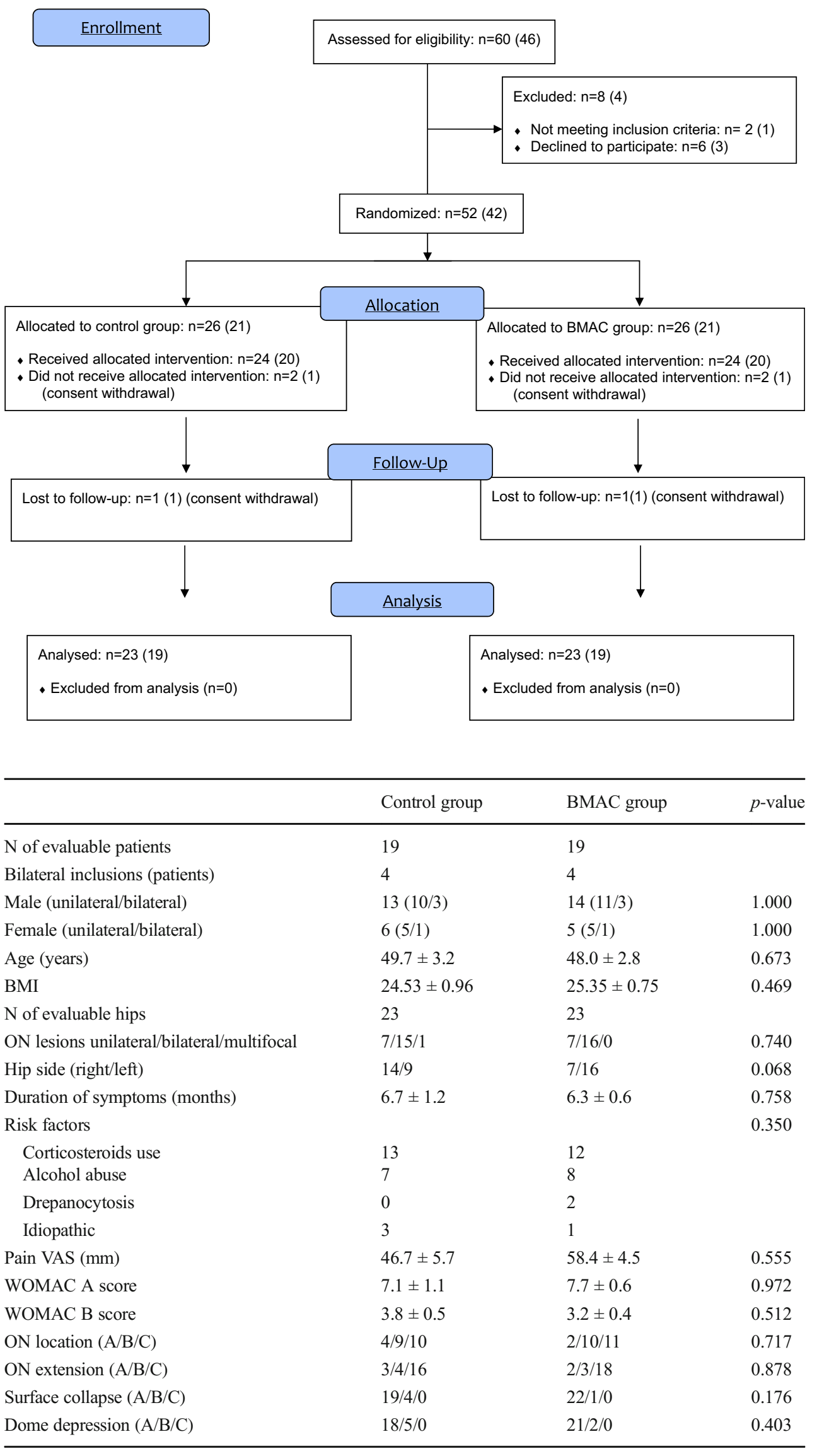

Table 1 Baseline demographic data and characteristics of ONFH 
Table 2 Results (hips)

\begin{tabular}{llll}
\hline & Control group & BMAC group & $p$-value \\
\hline N hips & 23 & 23 & \\
THR & 15 & 15 & 1.000 \\
Time to THR (month) & $8.2(2-12)$ & $9.7(6-22)$ & 0.382 \\
THR in bilateral ON yes/no & $7 / 1$ (stage 4) & $7 / 1$ (stage 4) & 1.000 \\
Evolution to stage 4 & 9 & 10 & 1.000 \\
Time to stage 4 (month) & $9.3(3-24)$ & $9.9(3.24)$ & 0.871 \\
To stage 4 without arthroplasty & 3 & 4 & 1.000 \\
Pain VAS evolution & $-2.3 \pm 6.4$ & $-7.7 \pm 5.9$ & 0.853 \\
WOMAC A score evolution & $-0.6 \pm 0.9$ & $-1.5 \pm 1.0$ & 0.678 \\
WOMAC B score evolution & $-0.1 \pm 0.4$ & $-1.5 \pm 1.3$ & 0.505 \\
ON location evolution $\mathrm{A} \rightarrow \mathrm{B} / \mathrm{A} \rightarrow \mathrm{C} / \mathrm{B} \rightarrow \mathrm{C}$ & $0 / 1 / 0$ & $0 / 0 / 2$ & 0.637 \\
ON extension evolution $\mathrm{A} \rightarrow \mathrm{B} / \mathrm{A} \rightarrow \mathrm{C} / \mathrm{B} \rightarrow \mathrm{C}$ & $0 / 0 / 1$ & $0 / 1 / 0$ & 1.000 \\
Surface collapse evolution $\mathrm{A} \rightarrow \mathrm{B} / \mathrm{A} \rightarrow \mathrm{C} / \mathrm{B} \rightarrow \mathrm{C}$ & $5 / 3 / 0$ & $6 / 4 / 0$ & 1.000 \\
Dome depression evolution $\mathrm{A} \rightarrow \mathrm{B} / \mathrm{A} \rightarrow \mathrm{C} / \mathrm{B} \rightarrow \mathrm{C}$ & $3 / 3 / 0$ & $3 / 1 / 3$ & 1.000 \\
\hline
\end{tabular}

Evolution $=$ difference between the baseline and the last values (24 months or THR timing)

THR was $9.7 \pm 1.2$ months for the BMAC group and $8.2 \pm 0.8$ months for the control group $(p=0.382)$.

The secondary objectives were to evaluate the advantage produced by BMAC concerning pain, functional ability, progression of $\mathrm{ON}$ lesions in terms of location, extension, surface collapse, dome depression evolution, and appearance of osteoarthritis features (evolution to ARCO stage 4). No significant improvement of pain (VAS) or functional ability (WOMAC subscales A and B) was found in the BMAC group. An evolution to stage 4 was noted in ten hips $(43 \%)$ in the BMAC group after $9.9 \pm 2.6$ months and nine hips $(43 \%)$ in the control group after $9.3 \pm 2.2$ months $(p=0.871)$. The $\mathrm{ON}$ lesions were only increased in a few hips (Table 2). Concerning the quantification of the $\mathrm{ON}$ lesions in the BMAC group versus control group, ON location was worsened in two hips $(9 \%)$ versus one $(5 \%)$, extension in one hip (5\%) versus one hip (5\%), surface collapse in ten hips (43\%) versus eight hips (38\%), and dome depression in seven hips $(30 \%)$ versus six hips $(29 \%)$. These ON lesion evolutions showed no statistical difference between the two groups ( $p$-values between 0.637 and $1.000)$ and were not related to the need of THR $(p=1.000)$.
The survey of potential side effects did not reveal any serious adverse event. Few adverse events were reported: pain at the great trochanter in four cases (one in the BMAC group) and at the iliac crest in two (two in the BMAC group), fever for less than 24 hours with negative bacteriological investigations in two cases in the BMAC group and nausea in two cases (one in the BMAC group).

\section{Discussion}

Our RCT has not confirmed the advantage of BMAC implantation in comparison with core decompression alone in $\mathrm{ON}$ stage 3. No statistically significant difference has been observed between the core decompression plus saline injection (control group) and the core decompression plus BMAC (implantation group) concerning the need for a THR, the evolution of pain and functional ability, the evolution of the necrotic lesion, and the appearance of osteoarthritis.

A review of the available literature revealed that no other RCT evaluating the effect of BMAC implantation or other cell-based treatments in stage $3 \mathrm{ONFH}$ was published. We
Table 3 Trials including stage 3 ONFH treated by core plus BMAc (see text)

\begin{tabular}{lllll}
\hline & Present study & Hernigou ref [13] & Wang ref [23] & Yoshioka ref [24] \\
\hline Trial design & RCT & Retrospective & Open prospective & Retrospective \\
Intervention & Mini-core & Mini-core & $2-3$ mini-core & Mini-core \\
$\mathrm{N}^{\circ}$ hip & 46 & 44 & 9 & 9 \\
Staging system & ARCO & Steinberg & ARCO & Japan Orthop Assoc \\
FU duration (month) & 24 & 92 & 28 & 41 \\
THR & $65 \%$ & $42 \%$ & $11 \%$ & $33 \%$ \\
\hline
\end{tabular}

$\mathrm{RCT}=$ randomized controlled trial. $\mathrm{THR}=$ total hip replacement. Mini core $=$ core with 3.5 to $5 \mathrm{~mm}$ outer diameter trephine 
only found publications where fractured ONFH were only a part of the studied cases (Table 3). Hernigou et al. reported a cohort study of ONFH treated with the same protocol as the one we used: mini core decompression and BMAC implantation in the necrotic area [13]. In this study, ON Steinberg stages 3 (no flattening of more than $1 \mathrm{~mm}$ ) and 4 (flattening of more than $1 \mathrm{~mm}$ without joint narrowing) were similar to our ON population although the definition of the staging was slightly different because we used the ARCO reference (no flattening more than $4 \mathrm{~mm}$ ). A second difference was the follow-up duration: more than five years in the Hernigou study versus two years in our study. Arthroplasty was needed in 5/12 ON stage 3 (42\%), and, in our study, in 15/23 hips $(65 \%)$. Some differences were found concerning the aetiological factors: more cases having alcohol abuse and sickle cell disease were present than in our study in which more patients had received corticosteroids. However, sickle cell disease seems to confer a worse ON prognosis [22]. So, the differences remained inexplicable. In another publication, a cohort study of BMAC treatment in ON included nine ARCO stage 3A ONFH with a follow-up of 27, six months [23]. The radiological progression occurred in 33, $3 \%$ and only $1 / 9$ required a THR. Finally, a retrospective study reporting nine hips in six SLE patients treated with corticoids included three Japanese orthopaedic stage 3 ONFH [24]. After a minimum follow-up of three years, $1 / 3$ hips had a radiological worsening and a THR requirement. These results are better than those of our study, but they are vitiated by some weaknesses: no control group and retrospective case reports.

Some limitations also exist in our study: many drop out reducing the studied cohort, the lack of sample size calculation, and the inclusion of bilateral ONFH. Absence of statistical effect does not however mean no effect as this could be attributed to too small sample sizes, but nevertheless no trend toward any difference was detected. So the rationale for further increasing the sample sizes has not been estimated relevant notably taking into account the difficulty of gathering the required patient's data.

Further regenerative therapy programs, using the RCT methodology, should further assess the therapeutic values of such programs including cell implantation, growth factors, and scaffolds.

In our study, the evolution of the ON lesions (extension, worsening of the subchondral bone collapse, evolution to osteoarthritis) was not correlated to the need of THR. In both groups the worsening of ON was very limited (Table 3). In ONFH, the need for THR could be related not only to a worsening of the collapse but also to other factors such as synovitis, cartilage lesions, or periarticular disturbances. Interestingly, a therapeutic approach of ONFH dedicated to mechanical stabilization of the collapse by cementation was ineffective [25]. A more precise analysis of lesion size based on the MRIs would confirm this lack of relationship between clinical failure and worsening of the bone collapse.

In conclusion, our study did not find any significant improvement of $\mathrm{ON}$ evolution in stage $3 \mathrm{ONFH}$ when BMAC implantation in the necrosis is added to core decompression.

Acknowledgments We thank Pierre Cullus of the Department of Biostatistics and Medical Informatics \& IRIBHM, School of Medicine, Université Libre de Bruxelles, for his contribution to data analyses.

Funding Funding was received from the Fund for Scientific Research of Belgium (FNRS) and the hospitals communities, University hospital of Liège and the Erasme hospital.

\section{Compliance with ethical standards}

Conflict of interest The authors declare no conflict of interest.

Ethical review committee statement All the authors confirm that the work described has not been published before; that it is not under consideration for publication anywhere else; that its publication has been approved by all coauthors, if any, as well as by the responsible authorities — tacitly or explicitly — at the institute where the work has been carried out.

Informed consent Informed consent was obtained from all individual participants included in the study.

The protocol was accepted by the ethical committees of both institutions. This trial was registered at ClinicalTrials.gov (ClinicalTrials.gov Identifier: NCT01544712).

\section{References}

1. Mont MA, Carbone JJ, Fairbank AC (1996) Core decompression versus nonoperative management for osteonecrosis of the hip. Clin Orthop Relat Res 324:169-178

2. Ficat P, Arlet J (1967) Ischémie et nécrose osseuse, exploration fonctionnelle de la circulation intra-osseuse et ses applications. Masson, Paris

3. Hauzeur JP, Malaise M, de Maertelaer V (2016) A prospective cohort study of the clinical presentation of non-traumatic osteonecrosis of the femoral head: spine and knee symptoms as clinical presentation of hip osteonecrosis. Int Orthop 40:1347-1351

4. Ficat P, Arlet J, Vidal R, Ricci A, Fournial JC (1971) Therapeutic results of drill biopsy in primary osteonecrosis of the femoral head (100 cases). Rev Rhum Mal Osteoartic 38:269-276

5. Koo KH, Kim R, Ko GH, Song HR, Jeong ST, Cho SH (1995) Preventing collapse in early osteonecrosis of the femoral head. A randomised clinical trial of core decompression. J Bone Joint Surg Br 77:870-874

6. Stulberg BN, Davis AW, Bauer TW, Levine M, Easley K (1991) Osteonecrosis of the femoral head. A prospective randomized treatment protocol. Clin Orthop Relat Res 268:140-151

7. Mont MA, Cherian JJ, Sierra RJ, Jones LC, Lieberman JR (2015) Nontraumatic osteonecrosis of the femoral head: where do we stand today? A ten-year update. J Bone Joint Surg Am 97:1604-1627

8. Hernigou P, Beaujean F, Lambotte JC (1999) Decrease in the mesenchymal stem-cell pool in the proximal femur in corticosteroidinduced osteonecrosis. J Bone Joint Surg Br 81:349-355

9. Gangji V, Hauzeur JP, Schoutens A, Hinsenkamp M, Appelboom T, Egrise D (2003) Abnormalities in the replicative capacity of 
osteoblastic cells in the proximal femur of patients with osteonecrosis of the femoral head. J Rheumatol 30:348-351

10. Mutijima E, De Maertelaer V, Deprez M, Malaise M, Hauzeur JP (2014) The apoptosis of osteoblasts and osteocytes in femoral head osteonecrosis: its specificity and its distribution. Clin Rheumatol 33:1791-1795

11. Gangji V, Hauzeur JP, Matos C, De Maertelaer V, Toungouz M, Lambermont M (2004) Treatment of osteonecrosis of the femoral head with implantation of autologous bone-marrow cells. A pilot study. J Bone Joint Surg Am 86-A:1153-1160

12. Gangji V, De Maertelaer V, Hauzeur JP (2011) Autologous bone marrow cell implantation in the treatment of non-traumatic osteonecrosis of the femoral head: Five year follow-up of a prospective controlled study. Bone 49:1005-1009

13. Hernigou P, Beaujean F (2002) Treatment of osteonecrosis with autologous bone marrow grafting. Clin Orthop Relat Res 405:14-23

14. Gangji V, Hauzeur JP (2005) Treatment of osteonecrosis of the femoral head with implantation of autologous bone-marrow cells. Surgical technique. J Bone Joint Surg Am 87(Suppl 1(Pt 1)):106112

15. Hauzeur JP, Pasteels JL, Schoutens A, Hinsenkamp M, Appelboom T, Chochrad I, Perlmutter N (1989) The diagnostic value of magnetic resonance imaging in non-traumatic osteonecrosis of the femoral head. J Bone Joint Surg Am 71:641-649

16. Mont MA, Marulanda GA, Jones LC, Saleh KJ, Gordon N, Hungerford DS, Steinberg ME (2006) Systematic analysis of classification systems for osteonecrosis of the femoral head. J Bone Joint Surg Am 88(Suppl 3):16-26

17. Muschler GF, Boehm C, Easley K (1997) Aspiration to obtain osteoblast progenitor cells from human bone marrow: the influence of aspiration volume. J Bone Joint Surg Am 79:1699-1709
18. Hauzeur JP, Orloff S, Taverne-Verbanck J, Pasteels JL (1986) Diagnosis of aseptic osteonecrosis of the femoral head by percutaneous transtrochanterian needle biopsy. Clin Rheumatol 5:346-358

19. Frank AJ, Moll JM, Hort JF (1982) A comparison of three ways of measuring pain. Rheumatol Rehabil 21:211-217

20. Bellamy N, Buchanan WW, Goldsmith CH, Campbell J, Stitt LW (1988) Validation study of WOMAC: a health status instrument for measuring clinically important patient relevant outcomes to antirheumatic drug therapy in patients with osteoarthritis of the hip or knee. J Rheumatol 15:1833-1840

21. Gardeniers JW (1992) A new international classification of osteonecrosis of the ARCO Committee on terminology and classification. J Jpn Orthop Assoc 66:18-20

22. Mont MA, Zywiel MG, Marker DR, McGrath MS, Delanois RE (2010) The natural history of untreated asymptomatic osteonecrosis of the femoral head: a systematic literature review. J Bone Joint Surg Am 92:2165-2170

23. Wang BL, Sun W, Shi ZC, Zhang NF, Yue DB, Guo WS, Xu SQ, Lou JN, Li ZR (2010) Treatment of nontraumatic osteonecrosis of the femoral head with the implantation of core decompression and concentrated autologous bone marrow containing mononuclear cells. Arch Orthop Trauma Surg 130:859-865

24. Yoshioka T, Mishima H, Akaogi H, Sakai S, Li M, Ochiai N (2011) Concentrated autologous bone marrow aspirate transplantation treatment for corticosteroid-induced osteonecrosis of the femoral head in systemic lupus erythematosus. Int Orthop 35:823-829

25. Gangji V, Rooze M, De Maertelaer V, Hauzeur JP (2009) Inefficacy of the cementation of femoral head collapse in glucocorticoidinduced osteonecrosis. Int Orthop 33:639-642 